\title{
Quantitative Analysis of Precipitate in Vanadium-microalloyed Medium Carbon Steels Using Small-angle X-ray and Neutron Scattering Methods
}

\author{
Yojiro OBA, ${ }^{1) *}$ Suresh KOPPOJU, ${ }^{1)}$ Masato OHNUMA, ${ }^{11}$ Toshio MURAKAMI, ${ }^{2,3)}$ Hitoshi HATANO, ${ }^{2,3)}$ \\ Kaoru SASAKAWA, ${ }^{4)}$ Amane KITAHARA ${ }^{4)}$ and Jun-ichi SUZUKI ${ }^{5}$ \\ 1) National Institute for Materials Science, Tsukuba, Ibaraki, 305-0047 Japan. \\ 2) Kobe Steel, Kobe, Hyogo, 651-2271 \\ Japan. $\quad 3)$ Consortium of the Japan Research and Development Center for Metals for Fundamental Studies on \\ Technologies for Steel Materials with Enhanced Strength and Functions, Tokyo, 105-0003 Japan. 4$)$ Kobelco Research \\ Institute, Inc., Kobe, Hyogo, 651-2271 Japan. $\quad$ 5) Japan Atomic Energy Agency, Tokai, Ibaraki, 319-1195 Japan.
}

(Received on April 25, 2011; accepted on August 8, 2011)

\begin{abstract}
The microstructures and chemical composition of nano-precipitates in vanadium (V) steels were investigated by the alloy contrast variation method (ACV) using small-angle X-ray scattering (SAXS) coupled with small-angle neutron scattering (SANS) at holding temperatures ranging between 600 and $700^{\circ} \mathrm{C}$. Both the SAXS and SANS profiles exhibited clear scattering, depending on the holding temperature, due to the presence of nano-precipitates. The scattering profiles of the precipitates are characteristic of spherical or disc-like particles. The average diameters of these precipitates increased from $0.5 \mathrm{~nm}$ at $600^{\circ} \mathrm{C}$ to $23 \mathrm{~nm}$ at $700^{\circ} \mathrm{C}$, whereas the number density of the precipitates decreases with increased holding temperature. Therefore, the increasing holding temperature results in an increase in the growth rate of the precipitates. ACV analysis revealed that the chemical composition of the precipitates corresponds to $\mathrm{NaCl}$-type vanadium carbide (VC) at 675 and $700^{\circ} \mathrm{C}$, and as $\mathrm{VC}_{0.9}$ at 625 and $650^{\circ} \mathrm{C}$. The formation of a different heterogeneity, non- $\mathrm{NaCl}$ type, was found in the sample at a holding temperature of $600^{\circ} \mathrm{C}$. This probably corresponds to a precursor of the $\mathrm{NaCl}$ phase in the initial process of precipitation.
\end{abstract}

KEY WORDS: heat treatment; alloying element; precipitation; phase analysis; small-angle scattering; SAXS; SANS.

\section{Introduction}

Steels are now required to have higher strength with lower alloying element content for lower energy consumption and $\mathrm{CO}_{2}$ emissions during production, transportation and use, and also for recyclability. Microalloyed steels with nano-size carbide are potential candidate materials fitting these requirements. In these alloys, however, the alloying elements which contribute to the strengthening of the steels by forming carbide or nitride are often rare metals. ${ }^{1,2)}$ Orowan's well-known model, precipitation hardening can be increased by decreasing the inter-precipitate-distance (and precipitate size) to the smallest limit of the dislocation by-passing mechanism. Therefore, precipitate size should be controlled to be as small as possible in order to obtain a higher precipitate number density per a certain volume fraction of precipitate. For this purpose, we need to know the mechanism of precipitation and the relationship between the process conditions and the resulting microstructures. In particular, acquisition of quantitative information of the microstructures, e.g., evolution of the shape, size, and number density, is required. In addition, it is also important to elucidate the chemical composition of the precipitates because it plays an important role in their kinetics.
Titanium, niobium and vanadium are well-known microalloying elements which often form MC-type carbides (M: metal) with a ferrite matrix of less than $10 \mathrm{~nm}^{2-4)}$ For vanadium, the fine structure has been intensively studied over the last few decades. Previous research shows that the crystal structure of the carbides is usually $\mathrm{NaCl}$-type, with a chemical composition of $\mathrm{VC}$ or $\mathrm{V}_{4} \mathrm{C}_{3}$, and the morphology of the precipitate is spherical, ellipsoid, needle, or plate, depending upon the composition and heat treatment. ${ }^{2,5-7)}$ Microstructural information was generally obtained using a transmission electron microscope (TEM). While this is the best tool to obtain qualitative microstructure information, the quantitative analysis of those vanadium steels is often insufficient to obtain representative statistical microstructure parameters because of limitations to the observed volume and low contrast in the coherent precipitates. Consequently, despite the volume of research, several topics are still controversial, e.g., the mechanism of evolution of precipitates, and even the chemical composition of the precipitates, especially during the early stages of precipitation. ${ }^{2}$ Compared to TEM, the information obtained from the small-angle scattering (SAS) technique is quite limited, but it is a complementary and suitable technique to obtain average and representative values for the size of the precipi- 
tates. $^{8-11)}$ However, few studies have used SAS on the microstructure of VC precipitates in steels. Recently, a new technique for quantitative analysis of the precipitates called alloy contrast variation (ACV) using small-angle X-ray (SAXS) and neutron scattering (SANS) was presented. ${ }^{12)}$ The information obtained from SAXS and SANS is basically identical, and reflects the size and shape of the precipitates. The difference between them is only in the scattering lengths of a material for an X-ray or a neutron. Since the scattering lengths are uniquely determined by the crystal structures (or mass density) and chemical compositions, it is possible to obtain information about the chemical composition of the precipitates using the ACV method, to complement the shape and size information obtained from the standard SAXS or SANS measurements. In addition, determination of the chemical composition provides a precise estimation of the number density of the precipitates.

In this study, ACV analyses of medium carbon vanadium steels using SAXS and SANS measurements are performed. The microstructures are quantitatively investigated, in addition to changes in the vanadium carbides during heat treatment, i.e., the effects of holding temperature.

\section{Experimental Procedure}

In order to examine the effect of vanadium, medium carbon steels with $\mathrm{V}$ and without vanadium were studied. The composition (in wt.\%) of the alloys is listed in Table 1. The alloys with $\mathrm{V}$ were heated at $1250^{\circ} \mathrm{C}$ and cooled at $20^{\circ} \mathrm{C} / \mathrm{s}$ to the holding temperatures $T(600,625,650,675$, and $700^{\circ} \mathrm{C}$ ) and then held for $1800 \mathrm{~s}$ in order to control the precipitation conditions of vanadium carbides, which were formed by interphase precipitation during ferrite and pearlite transformation. The matrices are mixed microstructures composed of ferrite and pearlite in the samples held at 600 , 625,650 , and $675^{\circ} \mathrm{C}$, and of ferrite, pearlite, and martensite in the sample held at $700^{\circ} \mathrm{C}$. The non-V sample was waterquenched from heating temperature to room temperature as a reference containing no vanadium precipitates.

The samples for the SAXS measurements were mechanically polished, typically to $20-30 \mu \mathrm{m}$, to achieve the appropriate transmission rate. Quantitative analysis of SAXS requires accurately known thickness at the measured position. To exclude the effects of thickness variation, the thickness of the samples was determined using the X-ray transmission rate and line absorption coefficient calculated from the chemical composition of the samples, and the massabsorption coefficients of the constituent element. ${ }^{12)}$ Using typical $\mathrm{Cu} K \alpha \mathrm{X}$-ray radiation, the SAXS measurement of steel is limited due to low transmission rates and high fluorescence. Hence, the SAXS measurements were performed using two fine-focus SAXS instruments with $\mathrm{Cr} K \alpha$ (Bruker, NANOSTAR) and Mo $K \alpha$ radiation (Rigaku Nano-Viewer) at the National Institute for Materials Science. Incident Xrays were focused using a Goebel mirror for Cr-SAXS, and

Table 1. Chemical composition of the samples (wt.\%).

\begin{tabular}{ccccccccc}
\hline & $\mathrm{C}$ & $\mathrm{Si}$ & $\mathrm{Mn}$ & $\mathrm{P}$ & $\mathrm{S}$ & $\mathrm{Al}$ & $\mathrm{V}$ & $\mathrm{N}$ \\
\hline non-V & 0.44 & 0.25 & 0.86 & 0.016 & 0.015 & 0.031 & 0 & 0.0036 \\
$\mathrm{~V}$ & 0.46 & 0.25 & 0.85 & 0.015 & 0.017 & 0.031 & 0.30 & 0.0042 \\
\hline
\end{tabular}

a two-dimensional confocal mirror for Mo-SAXS. In the Mo-SAXS experiments, the distances between the samples and the detector ( $\sim 350$ and $1100 \mathrm{~mm})$ were selected to cover a wide $q$ region $\left(0.2\right.$ to $\left.10 \mathrm{~nm}^{-1}\right)$. Here, the modulus of scattering vector, $q$, equals $4 \pi \sin \theta / \lambda$, where $\theta$ is half the scattering angle and $\lambda$ is the wavelength of the incident beam. The total $q$ range of the SAXS profiles was $0.07 \leq$ $q \leq 10 \mathrm{~nm}^{-1}$. For both instruments, a two-dimensional detector (Bruker HiSTAR) was used. The SAXS intensities were converted into absolute units using glassy carbon provided by the Argonne National Laboratory as a secondary standard. $^{13)}$

The samples for the SANS measurements were formed into an area of $2 \mathrm{~cm} \times 2 \mathrm{~cm}$ and a thickness of $2.0 \mathrm{~mm}$. SANS measurement was performed using the SANS-J-II installed in the JRR-3 reactor of the Japan Atomic Energy Agency. A wavelength of $0.65 \mathrm{~nm}$ with a resolution of $13 \%$ was selected using a velocity selector. A two-dimensional position-sensitive detector was used to obtain the scattering profiles. The distances between the samples and the detector were $\sim 2200 \mathrm{~mm}$ and $10000 \mathrm{~mm}$. The SANS $q$ range was between 0.03 and $1 \mathrm{~nm}^{-1}$. Detailed description of the instrumentation has been given in previous reports. ${ }^{14,15)}$ To separate the magnetic scattering contribution from the nuclear scattering, a magnetic field of $1.0 \mathrm{~T}$ was applied in the vertical direction. ${ }^{16)}$ Since the magnetization of the samples is completely saturated in this field, the scattering intensity parallel to the magnetic field includes only the nuclear contribution. Irradiated aluminum was measured as a secondary standard for the SANS intensity in absolute units. ${ }^{17)}$

\section{Results \& Discussion}

Figure 1 shows a typical bright-field TEM image of the V-containing steel with the holding temperature $T=700$. It can be seen from the micrograph that the shape of the precipitates are spherical or disc-like with the average size of $\sim 10 \mathrm{~nm}$. For the present sample composition, the thermal equilibrium calculation using the Thermo-calc indicates that the thermal equilibrium state of precipitate is an $\mathrm{NaCl}$-type vanadium carbide (VC). ${ }^{18)}$

The SAXS profiles observed from $\mathrm{V}$ and non-V-containing steels in absolute units are shown in Fig. 2. The intensity of the non- $\mathrm{V}$ sample is a featureless decreasing curve which originates from heterogeneities which can be inclusions

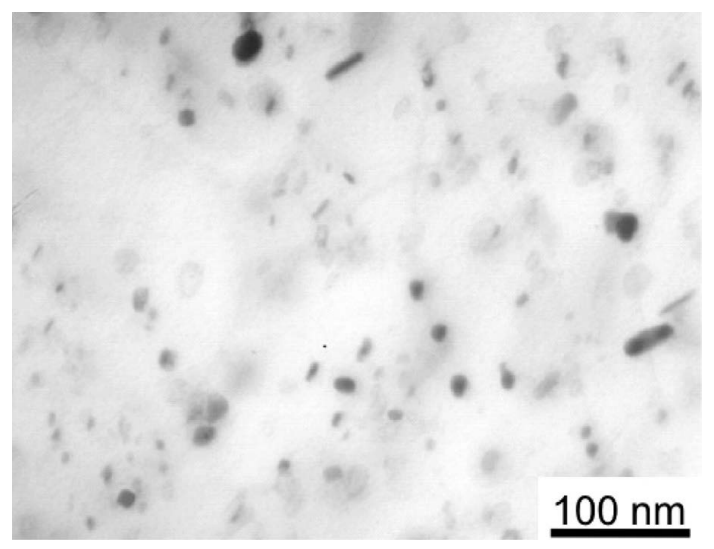

Fig. 1. Bright-field TEM image of the V steel with $T=700^{\circ} \mathrm{C}$. 


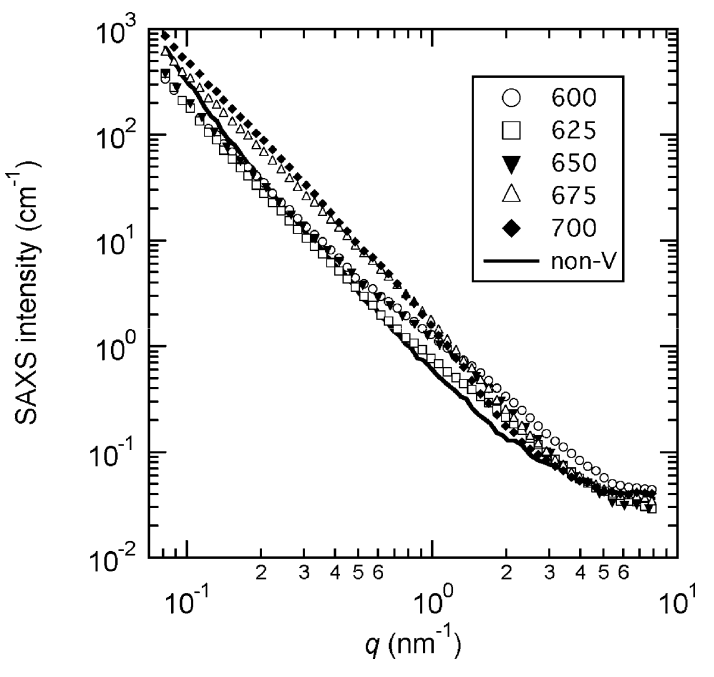

Fig. 2. SAXS profiles of the $V$ and non- $V$ steels.

$\left(\mathrm{MnS}, \mathrm{Al}_{2} \mathrm{O}_{3}\right.$, etc.) formed in the melting process, and is not related to vanadium compounds. The SAXS profile of the non-V sample does not show the clear plateau region of a Guinier regime. ${ }^{19)}$ Regarding the $q$-region where the Guinier approximation can be applied, this profile corresponds to the scattering from structures with a radius of gyration larger than $\sim 43 \mathrm{~nm}$, corresponding to the radius of a spherical particle larger than $\sim 55 \mathrm{~nm}$. In the other samples containing V, a weak shoulder is observed around $q \sim 1 \mathrm{~nm}^{-1}$. This is scattering from $\mathrm{V}$ compounds and reveals the formation of nanosize precipitates in all of the samples with $\mathrm{V}$.

For further analysis, the contribution of heterogeneity larger than the precipitates is an obstacle because it hides scattering features of the small precipitates, such as plateau region and $q^{-2}$ dependence. Since the SAXS profile of the non-V sample can be regarded as the contribution from only the larger heterogeneity, it was subtracted from the SAXS profiles of the other V samples as the background. ${ }^{9}$ Figure 3 shows the SAXS profiles from which that of the non-V sample was subtracted. All of the samples show clear scattering from the $\mathrm{V}$ precipitates. It can be clearly seen from Fig. 3 that with an increase in $T$, the Guinier region (plateau) is shifted toward the low $q$ region, which indicates that the size of the precipitates increases with increased $T$. The SAXS intensities of the samples held at higher $T$ (675 and $\left.700^{\circ} \mathrm{C}\right)$ show plateaus in the lowest $q$ region $\left(q<0.2 \mathrm{~nm}^{-1}\right)$. Then, they decrease in proportion to $q^{-2}$ in the middle $q$ region $\left(0.2<q<1 \mathrm{~nm}^{-1}\right)$, and fall off steeply in the highest $q$ region $\left(q>1 \mathrm{~nm}^{-1}\right)$. These features are consistent with the scattering of randomly-oriented disc particles. ${ }^{19,20)}$ In the other samples $\left(600,625\right.$, and $\left.650^{\circ} \mathrm{C}\right)$, the SAXS intensities show plateaus in the lower $q$ region, followed by a gradual reduction, and do not show a clear $q^{-2}$ dependence. These features indicate that the shape of the precipitates is disc or spherical, with a large size distribution. ${ }^{19,20)}$ These features in the shapes of the precipitates are consistent with the results of TEM observation and previous researches. ${ }^{5-7)}$

A curve fitting analysis was then carried out. Based on the TEM observation and the above discussion, two kinds of fitting functions were used. One is the small-angle scattering intensity $I(q)$ of randomly oriented disc particles described as:

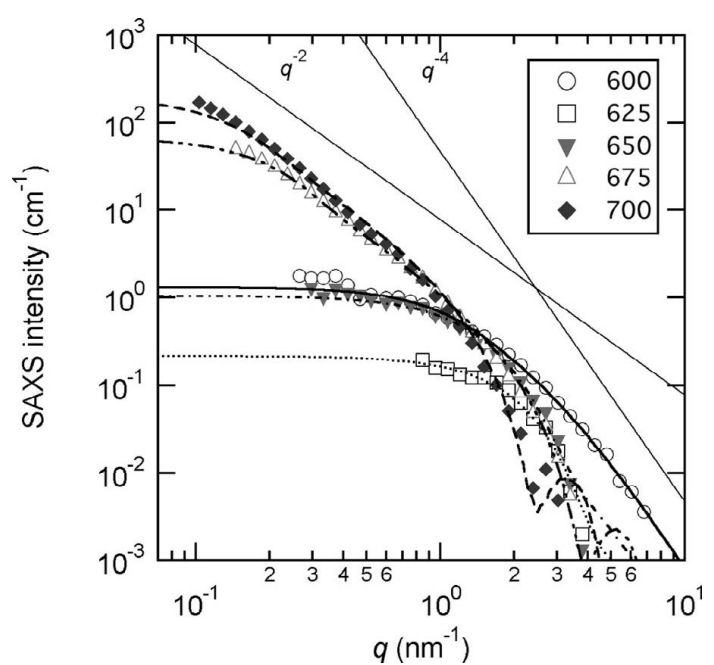

Fig. 3. Subtracted SAXS profiles of the V steels. The SAXS profile of the non-V steels was subtracted to extract the SAXS contributions of the nano-precipitates. Plots and solid lines are experimental data and fitted curves (Eqs. 1 and 4), respectively.

$$
I(q)=\Delta \rho^{2} d_{N} \int_{0}^{\infty} V_{\text {disc }}^{2}(r, t) F_{\text {disc }}^{2}(q, r, t) N(r) d r
$$

where $V(r), F(q, r, t)$, and $N(r)$ represent the volume, form factor and size distribution of precipitates, respectively, with a radius $r$ of the basal plane, and a thickness of the disc $t^{20)}$ $\Delta \rho$ is the difference in scattering length density between the matrix and the precipitates. $d_{N}$ is the number density of the precipitates. $F(q, r, t)$ and $V(r, t)$ are given by Eqs. (2) and (3):

$$
\begin{aligned}
& F_{d i s c}(q, r, t)=\int_{0}^{\pi / 2}\left[\frac{2 B_{1}(q r \sin \alpha)}{q r \sin \alpha} \frac{\sin (q t \cos / \alpha)}{q t \cos / \alpha}\right]^{2} \sin \alpha d \alpha \\
& V_{\text {disc }}(r, t)=\pi r^{2} t
\end{aligned}
$$

where $B_{1}(x)$ is the first-order Bessel function. The samples held at higher $T\left(675\right.$ and $\left.700^{\circ} \mathrm{C}\right)$ were analyzed using the above equations. On the other hand, it is difficult to obtain sufficient information from the 600,625 , and $650^{\circ} \mathrm{C}$ samples using the disc model, due to the unclear $q^{-2}$ dependence. Thus, another equation was used, the scattering from spherical particles, represented as:

$$
I(q)=\Delta \rho^{2} d_{N} \int_{0}^{\infty} V_{\text {sphere }}^{2}(r) F_{\text {sphere }}^{2}(q, r) N(r) d r
$$

where $V(r)$ and $F(q, r)$ are given by:

$$
\begin{array}{r}
F_{\text {sphere }}(q, r)=3 \frac{\sin (q r)-q r \cos (q r)}{(q r)^{3}} \ldots \ldots .(5), \text { and } \\
V_{\text {sphere }}=\frac{4}{3} \pi r^{3} \ldots \ldots \ldots \ldots \ldots \ldots \ldots \ldots \ldots . . .(6) .
\end{array}
$$

The size distribution of the precipitates in steel often follows a logarithmic normal function. We used the function described by:

$$
N(r)=\frac{1}{\sqrt{2 \pi} w r} \exp \left\{-\frac{\left(\ln r-\ln r_{m}\right)^{2}}{2 w^{2}}\right\} \ldots \ldots \ldots(7)
$$


Using the parameters $r_{\mathrm{m}}$ and $w$, the average precipitate diameter, $D_{\text {ave }}$, and the standard deviation, $\sigma$, of the particle diameter are expressed as:

$$
\begin{gathered}
D_{\text {ave }}=2 r_{m} \exp \left(w^{2} / 2\right) \ldots \ldots \ldots \\
\sigma=2 r_{m} \sqrt{\exp \left(w^{2}\right)\left\{\exp \left(w^{2}\right)-1\right\}} .
\end{gathered}
$$

To simplify the analyses, $t$ is regarded as a constant without size distribution in this study.

Figure 3 also shows the results of fitting. The fitted curves agree well with the corresponding experimental data. The parameters obtained, $D_{\text {ave }}, \sigma$, and $t$, are shown in Fig. 4. These clearly show that the size of the precipitates increases with increasing holding temperature. The average diameter of the precipitates increases from $0.5 \mathrm{~nm}$ at $600^{\circ} \mathrm{C}$ to $23 \mathrm{~nm}$ at $700^{\circ} \mathrm{C}$. This tendency, as well as the range of sizes, is close to the values reported in a previous study. ${ }^{\text {) }}$

The SANS profiles of the same samples are shown in Fig. 5. The scattering in the low $q$ region shows almost the same behavior in all samples. In the $q$ region higher than $0.3 \mathrm{~nm}^{-1}$, the SANS profiles become different from each other, and increase in the samples with $\mathrm{V}$ compared with the non-V sample. These profiles are composed of the background contribution and scattering from the precipitates, as seen in the SAXS profiles. In order to exclude the background contribution, the SANS profile of the non-V sample was subtracted from the profiles of the other samples in the same manner as in the SAXS analyses. Figure 6 shows the background-subtracted SANS profiles. Although they basically include the scattering contribution only from the precipitates, in the $q<0.6 \mathrm{~nm}^{-1}$ of the samples 650,675 and 700, the deviation of the SANS profiles from the SAXS profiles is observed. This probably reflects the difference in the heterogeneity not related with the vanadium compounds larger
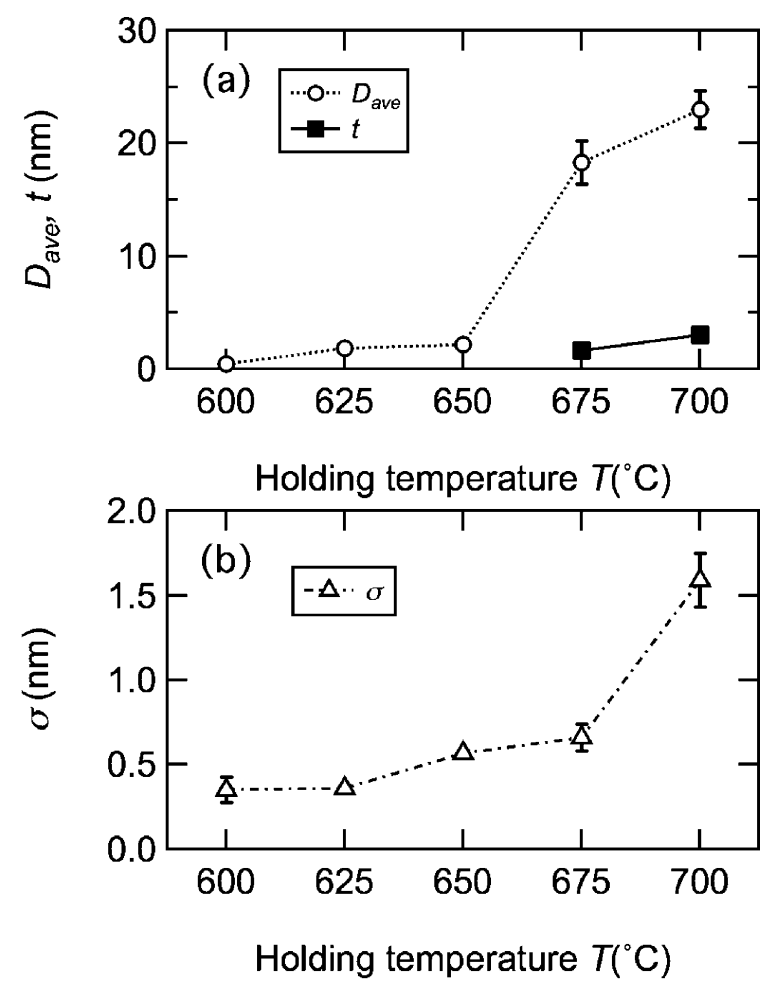

Fig. 4. $T$ dependence of (a) $D_{\text {ave }}$ and $t$; and (b) $\sigma$. than the precipitates. Since the scattering of this heterogeneity is not clearly observed in the corresponding $q$ range of the SAXS profiles, its candidate compound is cementite particles. The $\Delta \rho_{x}$ of the cementite in ferrite matrix is negligibly low, while its $\Delta \rho_{n}$ is significantly high. ${ }^{21)}$ Based on the Porod law, this contribution rapidly decreases in proportion to $q^{-4}$ in high $q$ region. Thus, in the $q>0.7 \mathrm{~nm}^{-1}$, the SANS profiles are regarded as the scattering mainly from the $\mathrm{V}$ precipitates and the analysis similar to the SAXS can be applied to the SANS. The observed $q$ range of the SANS profiles is insufficient for a curve fitting analysis with completely free parameters of size, e.g., $r$ and $t$ in the above equations. However, since the origin of SANS intensities is intrinsically identical to that of the SAXS intensities except for a difference between $\Delta \rho_{x}$ and $\Delta \rho_{n}$, the size parameters must be the same between SAXS and SANS. Hence, when the parameters $r$ and $t$ are fixed using the results of the SAXS analysis, it is possible to carry out the curve-fitting analysis. The results of the fitting analyses of the SAXS and SANS measurements give the ratio of the square of the scat-

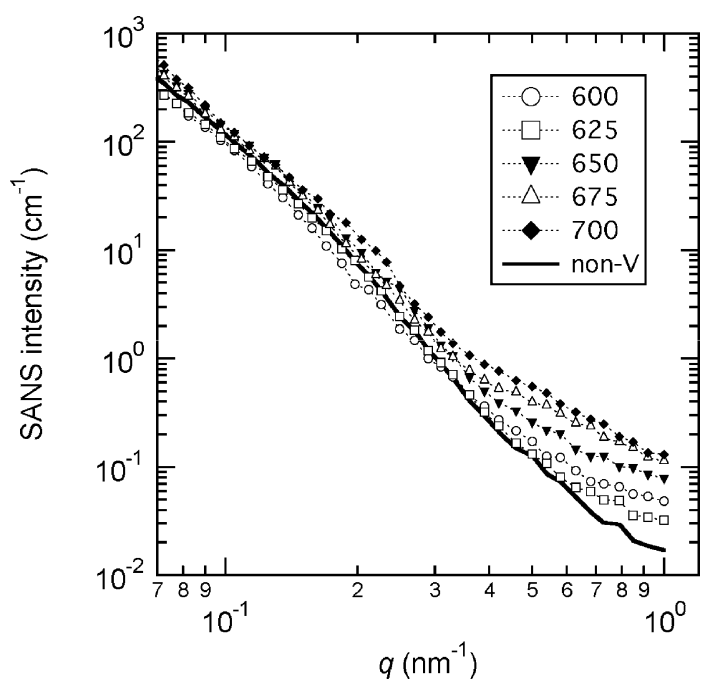

Fig. 5. SANS profiles of $\mathrm{V}$ and non- $\mathrm{V}$ steels.

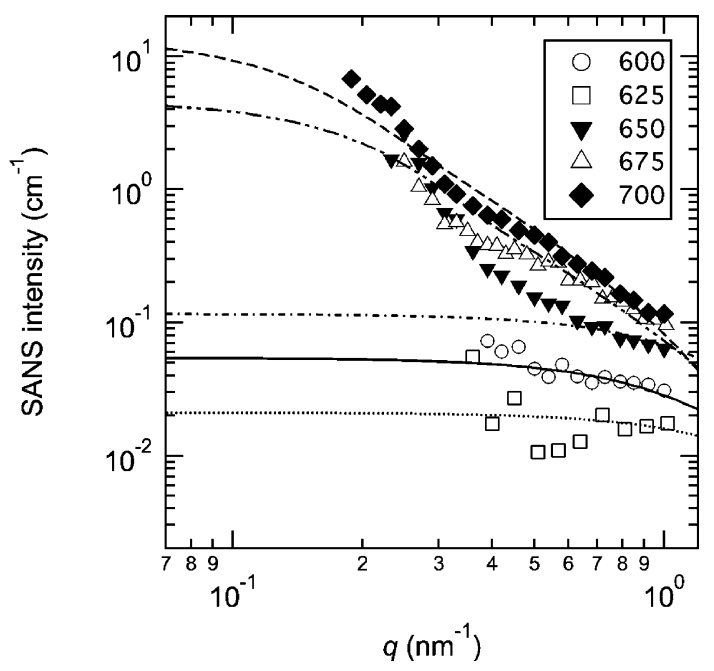

Fig. 6. Subtracted SANS profiles of the V steels. Similar to the SAXS profiles, they coincide with the contributions of the precipitates only. Plots and solid lines are experimental data and fitted curves (Eqs. 1 and 4), respectively. 
tering length density difference $\Delta \rho_{x}{ }^{2} / \Delta \rho_{n}{ }^{2}{ }^{12)}$ Since the values of $\Delta \rho_{x}^{2} / \Delta \rho_{n}^{2}$ are dependent merely upon the crystal structures and chemical composition of the precipitates, each compound has a unique value. Figure 7 shows $\Delta \rho_{x}^{2} /$ $\Delta \rho_{n}{ }^{2}$ as a function of $T$. The values of $\Delta \rho_{x}^{2} / \Delta \rho_{n}{ }^{2}$ can be roughly sorted into 3 categories: the highest value at $600^{\circ} \mathrm{C}$, the lowest value at 625 and $650^{\circ} \mathrm{C}$, and the middle value at 675 and $700^{\circ} \mathrm{C}$. Since the experimental error of $\Delta \rho_{x}{ }^{2} / \Delta \rho_{n}{ }^{2}$ was estimated to be about $\pm 10 \%,{ }^{12)}$ the difference obtained is significant, and shows that the precipitation occurs via 3 processes.

For compounds with well-defined crystal structures and chemical composition, the value of $\Delta \rho_{x}^{2} / \Delta \rho_{n}^{2}$ can also be evaluated from that data. ${ }^{22)}$ According to previous reports, ${ }^{23-25)}$ $\mathrm{NaCl}$-type $\mathrm{VC}_{x}$ with a varying fraction of carbon ( $x$ value) is the first candidate for the precipitate in $\mathrm{V}$ steels in this temperature and size range. Figure 8 shows the values of $\Delta \rho_{x}^{2} / \Delta \rho_{n}^{2}$ calculated from the chemical composition and crystal structures using literature data. There is a linear increase in $\Delta \rho_{x}^{2} / \Delta \rho_{n}^{2}$ with an increase in $x$ from 0.72 to 0.94 .

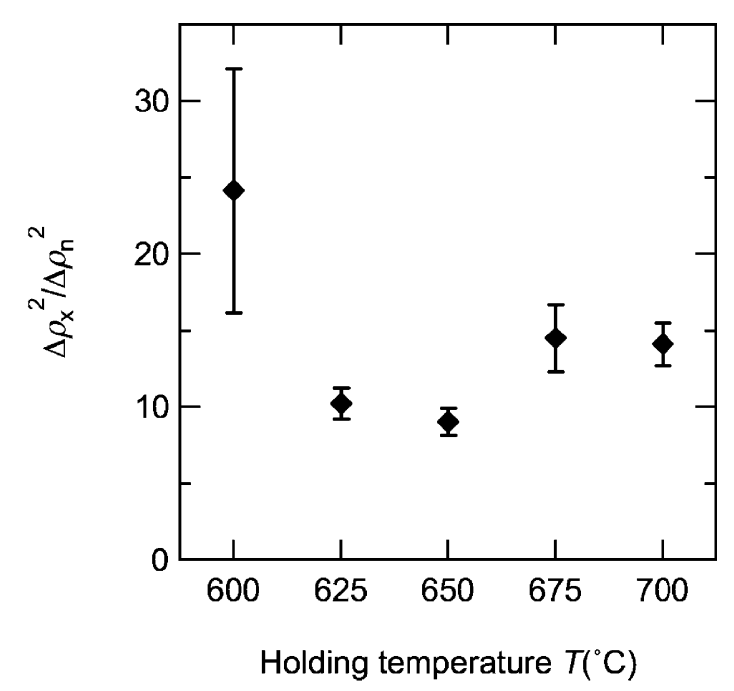

Fig. 7. $\Delta \rho_{x}{ }^{2} / \Delta \rho_{n}{ }^{2}$ estimated from experimental results as a function of $T$. The values at 675 and $700^{\circ} \mathrm{C}$ are almost equal, within the error. The values at 625 and $650^{\circ} \mathrm{C}$ are lower than this, and the value at $600^{\circ} \mathrm{C}$ is higher.

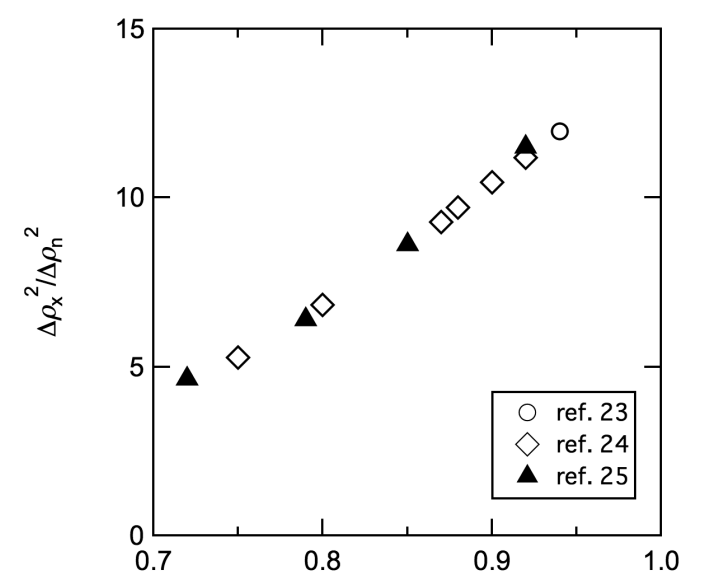

Chemical composition of carbon $x$ in $\mathrm{NaCl}$-type $\mathrm{VC}_{x}$

Fig. 8. $\Delta \rho_{x}^{2} / \Delta \rho_{n}^{2}$ values calculated from the crystal structures and chemical composition-as a function of $x .^{3-5)}$
The middle $\left(675\right.$ and $\left.700^{\circ} \mathrm{C}\right)$ and highest values $(625$ and $650^{\circ} \mathrm{C}$ ) obtained from the experiments are located in this region. The lowest value corresponds to the value calculated at $x=0.9$, while the middle value is slightly higher than that at $x=0.94$. This indicates that at $T=625$ and $650^{\circ} \mathrm{C}$, the precipitates have a carbon deficient structure, while at $T=$ 675 and $700^{\circ} \mathrm{C}$, the chemical composition of the precipitates is almost perfect $\mathrm{VC}_{1.0}$. Thus, we conclude that equal to and above $625^{\circ} \mathrm{C}, \mathrm{NaCl}$-type $\mathrm{VC}_{x}$ nanoparticles are formed in the steels. Ishiguro and Sato also have reported nonstoichiometric $\mathrm{MC}_{x}$ in ultra-low carbon steels. ${ }^{26)}$ Using electron energy loss spectroscopy (EELS) in a field-emission TEM, $\mathrm{TiC}_{x}$ and $(\mathrm{Ti}, \mathrm{Nb}) \mathrm{C}_{x}$ were observed. The values of $x$ were $0.82-0.91$ in the Ti- and Ti-Nb-bearing steels. This range of $x$ is consistent with our results. On the other hand, the $\Delta \rho_{x}^{2} / \Delta \rho_{n}^{2}$ at $T=600^{\circ} \mathrm{C}$ does not agree with the values calculated for $\mathrm{VC}_{x}$. This compound probably corresponds to the initial processes of precipitation on the nanometer scale. Taking their size into account, it seems that clusters or embryos with different crystal structures from VC are being detected. To identify the structure, further studies with TEM or other techniques are necessary.

In the $625-700^{\circ} \mathrm{C}$ samples, since the chemical composition of the precipitates was identified, $d_{N}$ can be evaluated from the results of the fitting analysis using the calculated $\Delta \rho_{x}$ or $\Delta \rho_{n}$ of each chemical composition along with Eq. (1). For the $600^{\circ} \mathrm{C}$ sample, since the phase was not completely determined, a possible range of $d_{N}$ was evaluated between $\mathrm{VC}_{0.72}$ and $\mathrm{VC}_{0.94}$ as a reference. Figure 9 shows $d_{N}$ as a function of $T$. This indicates that a remarkably large amount of precipitate is formed even at $600^{\circ} \mathrm{C}$. Above $600^{\circ} \mathrm{C}$, the amount tends to decrease with increasing $T$. The magnitude of $d_{N}$ is between $\sim 10^{17} \mathrm{~cm}^{-3}$ at $625^{\circ} \mathrm{C}$ and $\sim 10^{15} \mathrm{~cm}^{-3}$ at $700^{\circ} \mathrm{C}$. This is interpreted as an increase in the precipitate growth rate with a rise in temperature. The volume fraction $V_{f}$ was then also estimated from $d_{N}$ and the average volume of the precipitate obtained from the results of the fitting analyses, $D_{\text {ave }}$ and $\sigma$, except for the $600^{\circ} \mathrm{C}$ sample (Fig. 10). $V_{f}$ reveals the increasing and saturation behavior with increasing $T$. The increasing behavior signifies that a higher

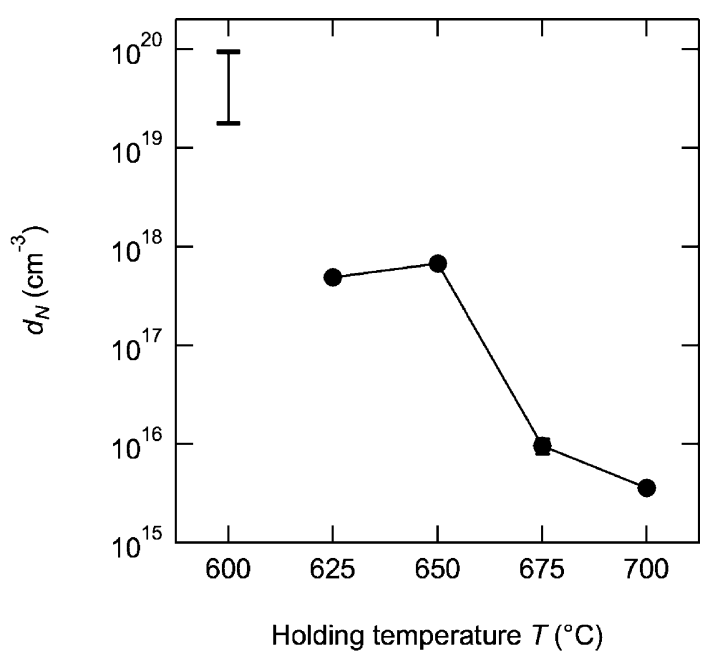

Fig. 9. Relationship between $d_{N}$ and $T$. At $600^{\circ} \mathrm{C}$, a possible range of $d_{N}$ estimated under the assumption that the precipitates are $\mathrm{NaCl}$-type $\mathrm{VC}_{x}$ with $x=0.72-0.94$ is shown for reference. 


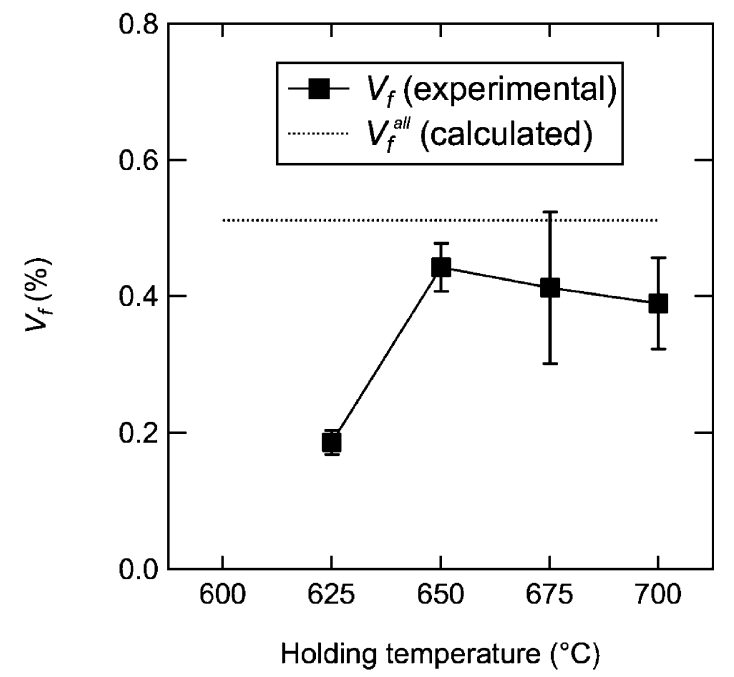

Fig. 10. Relationship between $V_{f}$ and $T$. The broken line indicates $V_{f}^{\text {all. }}$.

holding temperature enhances precipitation growth. Fig. 10 also shows the maximum values of volume fraction, $V_{f}^{\text {all }}$, calculated from the chemical composition under the assumption that all $\mathrm{V}$ was completely precipitated as $\mathrm{NaCl}$ type $\mathrm{VC}_{1.0}$. The saturation values of $V_{f}$ are close to $V_{f}^{\text {all }}$. Hence, almost all $\mathrm{V}$ is precipitated as $\mathrm{VC}$ particles between $T=650$ and $700^{\circ} \mathrm{C}$, while at $625^{\circ} \mathrm{C}$, a portion of the $\mathrm{V}$ is still diffused in the matrix.

The hardness of the samples is shown in Fig. 11. For the hardness measurements, the non-V samples with holding temperatures $T=600,650$, and $700^{\circ} \mathrm{C}$ were also prepared for comparison. The non- $\mathrm{V}$ sample shows an increase in hardness at $700^{\circ} \mathrm{C}$. For this sample, the martensite formed in the matrix gives rise to the hardening. At $T$ equals 600 and $650^{\circ} \mathrm{C}$, the samples with $\mathrm{V}$ are significantly harder than the non-V samples. This is attributed to precipitation hardening. Note that the hardening occurs even in the $600^{\circ} \mathrm{C}$ sample, even though the phase of the precipitate in this sample is likely not the $\mathrm{NaCl}$-type $\mathrm{VC}_{x}$, which shows that precipitates other than $\mathrm{VC}_{x}$ also contribute to the hardening. Despite the lower $d_{N}$ compared with the $650^{\circ} \mathrm{C}$ sample, the $700^{\circ} \mathrm{C}$ sample still shows higher hardness. At this temperature, hardening due to martensite is probably dominant.

The effect of the precipitates on the mechanical properties is generally described by Ashby-Orowan equation: ${ }^{27,28)}$

$$
\Delta Y S=m \cdot 0.84\left(\frac{1.2 G b}{2 \pi L}\right) \cdot \ln \left(k \frac{x}{2 b}\right)
$$

where $\triangle Y S$ is the increment in yield strength, $m$ is the Taylor factor. $G$ and $b$ are the shear modulus and the Burgers vector, respectively. $L$ is the distance between two precipitates on the slip plane, $k$ is the correction factor about the interaction between dislocations. $G$ and $b$ are $75 \mathrm{GPa}^{29)}$ and $0.25 \mathrm{~nm}$ for the medium carbon steel. For vanadium microallyed medium carbon steels, $k$ is 2.7 in ferrite and 1.0 in pearlite, and $m$ is $2.0 .^{27)} x$ is the particle diameter on the slip plane and described as follows when the precipitates are regarded as the randomly dispersed spherical particles:

$$
x=\sqrt{\frac{\pi}{4}} D_{\text {ave }}
$$

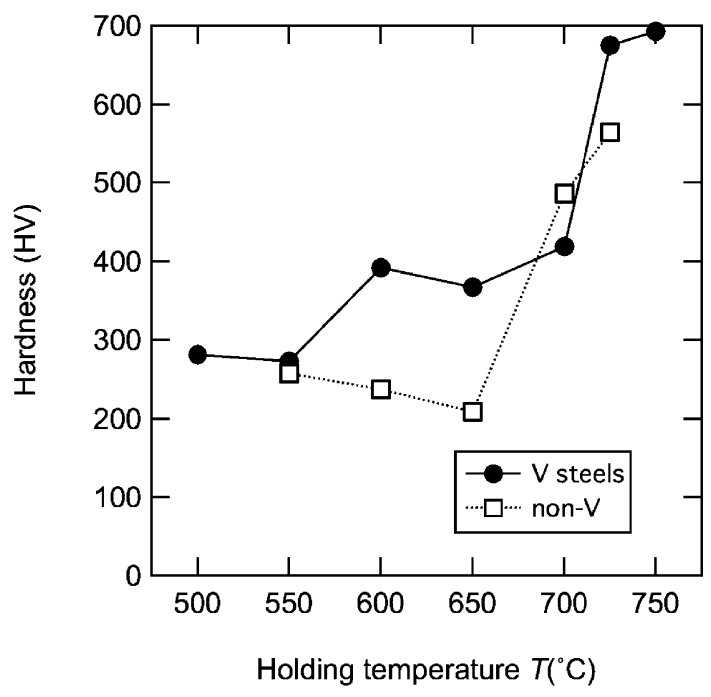

Fig. 11. Relationship between hardness and $T$.

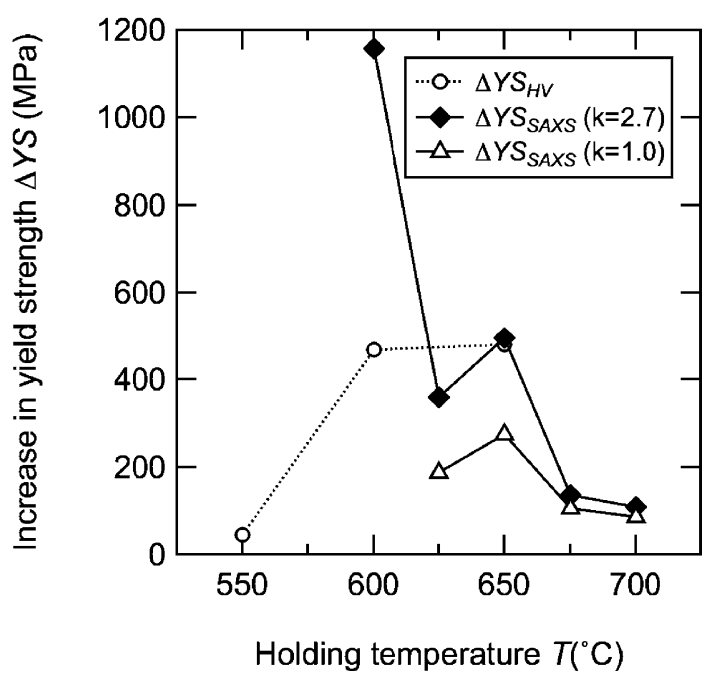

Fig. 12. $T$ dependence of $\Delta Y S_{\mathrm{SAXS}}$ and $\Delta Y S_{\mathrm{HV}}$.

$L$ is expressed as:

$$
L=\frac{D_{\text {ave }}}{2} \sqrt{\frac{2 \pi}{3 V_{f}}}-x
$$

Substituting Eqs. (10) and (11) and the parameters obtained from the SAXS for Eq. (9) gives the $\Delta Y S$ ( $\left.\triangle Y S_{\mathrm{SAXS}}\right)$. Since the hardness $H$ is empirically expressed as $\triangle Y S(\mathrm{MPa})=$ $3.04 H(\mathrm{HV}){ }^{30)} \Delta Y S$ can be also evaluated from the difference in the hardness between $\mathrm{V}$ samples and non- $\mathrm{V}$ samples at 550,600 and $650^{\circ} \mathrm{C}\left(\Delta Y S_{\mathrm{HV}}\right)$. Figure 12 shows $\Delta Y S_{\mathrm{SAXS}}$ and $\Delta Y S_{\mathrm{HV}}$ as a function of $T$. At 625 and $650^{\circ} \mathrm{C}$, while the values of $\Delta Y S_{\mathrm{SAXS}}$ result in underestimation for $k=1.0$, they are consistent with $\Delta Y S_{\mathrm{HV}}$ for $k=2.7$. This indicates that the precipitation strengthening in the ferrite has a dominant influence on the hardness. At 675 and $700^{\circ} \mathrm{C}$, the values of $\Delta Y S_{\text {SAXS }}$ decrease to $\sim 30 \%$. Although the contribution of the precipitates is not clear in the hardness measurements due to the formation of the martensite, $\triangle Y S_{\mathrm{SAXS}}$ reveals that the precipitation strengthening occurs and is reduced probably caused by coarsening of the precipitates. At $600^{\circ} \mathrm{C}, \Delta Y S_{\mathrm{SAXS}}$ for $k=2.7$ is distinctly higher than $\Delta Y S_{\mathrm{HV}}$. This is probably attributed to the less contribution of the small precipitates in which the Ashby-Orowan mechanism is not accepted, as 
well as the uncertainty of the chemical composition and number density at $600^{\circ} \mathrm{C}$.

\section{Conclusion}

In conclusion, the fine structures of vanadium carbides precipitated in medium carbon steels and the changes therein with varying holding temperature were investigated using SAXS and SANS measurements and ACV analysis. The results are as follows:

(1) Nanosize precipitates appear for $T=600$ to $700^{\circ} \mathrm{C}$.

(2) The SAXS profiles indicate that the shape of the precipitates is spherical or disc-like below and equal to $650^{\circ} \mathrm{C}$, and disc-like over $650^{\circ} \mathrm{C}$.

(3) The chemical composition of the precipitates is NaCl-type VC at $T=675$ and $700^{\circ} \mathrm{C}$, and carbon deficient $\mathrm{VC}_{x}(\mathrm{x} \sim 0.9)$ at $T=625$ and $650^{\circ} \mathrm{C}$. At $600^{\circ} \mathrm{C}$, the chemical composition of the precipitate is not $\mathrm{NaCl}$-type $\mathrm{VC}_{x}$. Taking their size into account, this indicates that clusters or embryos having a different crystal structure from $\mathrm{VC}_{x}$ are formed in an initial precipitation process.

(4) With an increase in $T$, the number density of the precipitates reduces from $\sim 10^{17} \mathrm{~cm}^{-3}$ at $625^{\circ} \mathrm{C}$ to $\sim 10^{15} \mathrm{~cm}^{-3}$ at $700^{\circ} \mathrm{C}$.

(5) Hardening occurs even in the $600^{\circ} \mathrm{C}$ sample in which the chemical composition of the precipitate is different from that of vanadium carbide.

\section{Acknowledgments}

The authors are grateful to Ms. Y. Kinjo for her help with the SANS measurements. Part of this work was carried out as a part of "Fundamental Studies on Technologies for Steel Materials with Enhanced Strength and Functions" by Consortium of the Japan Research and Development Center for Metals. Financial support from NEDO is gratefully acknowledged.

\section{REFERENCES}

1) D. A. Porter and K. E. Easterling: Phase Transformations in Metals and Alloys, Chapman \& Hall, London, (1981), 417.

2) T. N. Baker: Mater. Sci. Technol., 25 (2009), 1083.

3) F. G. Wei and K. Tsuzaki: Metall. Mater. Trans. A, 37A (2006), 331.

4) H. R. H. Bajguirani: Mater. Sci. Eng., A338 (2002), 142.

5) R. G. Baker and J. Nutting: Precipitation processes in steels, Special report no. 64, The Iron and Steel Institute, London, (1959), 1.

6) E. Smith and J. Nutting: J. Iron Steel Inst., 187 (1957), 314.

7) A. K. Seal and R. W. K. Honeycombe: J. Iron Steel Inst., 188 (1958), 9.

8) H. Yasuhara, K. Sato, Y. Toji, M. Ohnuma, J. Suzuki and Y. Tomota: Tetsu-To-Hagané, 96 (2010), 545.

9) M. Ojima, M. Ohnuma, J. Suzuki, S. Ueta, S. Narita, T. Shimizu and Y. Tomota: Scr. Mater., 59 (2008), 313.

10) M. Große, A. Gokhman and J. Böhmert: Nucl. Instrum. Methods Phys. Res. B, 160 (2000), 515.

11) A. J. Allen, D. Gavillet and J. R. Weertman: Acta Metall. Mater., 41 (1993), 1869.

12) M. Ohnuma, J. Suzuki, S. Ohtsuka, S.-W. Kim, T. Kaito, M. Inoue and H. Kitazawa: Acta Mater., 57 (2009), 5571.

13) F. Zhang, J. Ilavsky, G. Long, J. Quintana, A. Allen and P. Jemian: Metall. Mater. Trans. A, 41A (2010), 1151.

14) H. Iwase, S. Koizumi, J. Suzuki, T. Oku, H. Sasao, H. Tanaka, H. M. Shimizu and T. Hashimoto: J. Appl. Cryst., 40 (2007), s414.

15) S. Koizumi, H. Iwase, J. Suzuki, T. Oku, R. Motokawa, H. Sasao, H. Tanaka, D. Yamaguchi, H. M. Shimizu and T. Hashimoto: J. Appl. Cryst., 40 (2007), s474.

16) G. L. Squires: INTRODUCTION TO THE THEORY OF THERMAL NEUTRON SCATTERING, Dover Publications, New York, (1996), 129.

17) G. D. Wignall and F. S. Bates: J. Appl. Cryst., 20 (1987), 28.

18) B. Sundman, B. Jansson and J.-O. Andersson: Calphad, 9 (1985), 153.

19) L. A. Feigin and D. I. Svergun: Structure Analysis by Small-Angle X-ray and Neutron Scattering, Plenum Press, New York, (1987), 59.

20) J. S. Pedersen: Adv. Colloid Int. Sci., 70 (1997), 171.

21) M. Ohnuma: J. JSNDI, 60 (2011), 86.

22) H. Rauch and W. Waschkowski: NEUTRON DATA BOOKLET, ed. by A.-J. Dianoux and G. Lander, Old City Publishing, Philadelphia, (2003), 1.1-1.

23) E. Rudy, F. Benesovsky and E. Rudy: Monatsh. Chem., 93 (1962), 693.

24) G. Brauer and W.-D. Schnell: J. Less Common Met., 7 (1964), 23.

$25)$ O. N. Carlson, A. H. Ghaneya and J. F. Smith: Phase Diagrams of Binary Vanadium Alloys, ed.by J. F. Smith, ASM Int., Ohio, (1989), 33.

26) Y. Ishiguro and K. Sato: Mater. Trans., JIM, 37 (1996), 643.

27) M. Umemoto, K. Sugita and H. Kuo: Proc. of Symp. on Controlled Forging Group in "Fundamental Studies on Technologies for Steel Materials with Enhanced Strength and Functions", NEDO, Tokyo, (2010), 9.

28) M. F. Ashby: Acta Metall., 14 (1966), 679.

29) T. Ohta, Y. Tsuchida, Y. Takeuti, K. Kodera and H. Nyuko: J. J.S.M.E., 71 (1968), 1496.

30) H. Yada: Prediction and Control of Microstructures and Mechanical Properties in Hot Strip Rolling, ISIJ, Tokyo, (1989), 172. 